\title{
The WNT signalling pathway and diabetes mellitus
}

\author{
T. Jin
}

Received: 17 March 2008 / Accepted: 16 May 2008 / Published online: 12 August 2008

(C) The Author(s) 2008

\begin{abstract}
The WNT signalling pathway is involved in many physiological and pathophysiological activities. WNT ligands bind to Frizzled receptors and co-receptors (LDL receptor-related protein 5/6), triggering a cascade of signalling events. The major effector of the canonical WNT signalling pathway is the bipartite transcription factor $\beta$-catenin/T cell transcription factor ( $\beta$-cat/TCF), formed by free $\beta$-cat and one of the four TCFs. The WNT pathway is involved in lipid metabolism and glucose homeostasis, and mutations in LRP5 may lead to the development of diabetes and obesity. $\beta$-Cat/TCF is also involved in the production of the incretin hormone glucagon-like peptide-1 in the intestinal endocrine L cells. More recently, genome-wide
\end{abstract}

T. Jin

Department of Medicine, University of Toronto,

Toronto, ON, Canada

T. Jin

Department of Physiology, University of Toronto,

Toronto, ON, Canada

T. Jin

Department of Laboratory Medicine and Pathobiology,

University of Toronto,

Toronto, ON, Canada

T. Jin $(\bowtie)$

10-354, Toronto Medical Discovery Tower,

the MaRS Building, Division of Cell and Molecular Biology,

Toronto General Research Institute,

University Health Network,

101 College Street,

Toronto, ON, Canada M5G 1L7

e-mail: tianru.jin@utoronto.ca association studies have identified $T C F 7 L 2$ as a diabetes susceptibility gene, and individuals carrying certain $T C F 7 L 2$ single nucleotide polymorphisms could be more susceptible to the development of type 2 diabetes. Furthermore, $\beta$-cat is able to interact with forkhead box transcription factor subgroup O (FOXO) proteins. Since FOXO and TCF proteins compete for a limited pool of $\beta$-cat, enhanced FOXO activity during ageing and oxidative stress may attenuate WNT-mediated activities. These observations shed new light on the pathogenesis of type 2 diabetes as an age-dependent disease.

Keywords $\beta$-cat/TCF. FOXO - GLP-1 - Stress .

TCF7L2 $\cdot$ WNT

$\begin{array}{ll}\text { Abbreviations } \\ \beta \text {-cat } & \beta \text {-catenin } \\ \text { CK-1 } \alpha & \text { casein kinase-1 } \alpha \\ \text { Dvl } & \text { Dishevelled protein } \\ \text { FOXO } & \text { forkhead box transcription factor subgroup O } \\ \text { GLP-1 } & \text { glucagon-like peptide-1 } \\ \text { GSK-3 } & \text { glycogen synthase kinase-3 } \\ \text { GWA } & \text { genome-wide association } \\ \text { LEF-1 } & \text { lymphoid enhancer-binding factor } 1 \\ \text { LRP } & \text { LDL receptor-related protein } \\ \text { LUC } & \text { luciferase } \\ \text { Pdx-1 } & \text { pancreas/duodenum homeobox protein 1 } \\ \text { PI3K } & \text { phosphatidylinositol 3-kinase } \\ \text { PKB } & \text { protein kinase B } \\ \text { ROS } & \text { reactive oxygen species } \\ \text { SNP } & \text { single nucleotide polymorphism } \\ \text { TCF } & \text { T cell factor }\end{array}$




\section{Glossary}

- $\beta$-cat/TCF This bipartite transcription factor functions as the major effect of the canonical WNT signalling pathway. It is formed by free $\beta$-catenin ( $\beta$-cat) and a $T$ cell factor (TCF)

- FOXO Forkhead box transcription factors subgroup $\underline{\mathrm{O}}$ are mediators of stress. FOXO proteins compete with TCF proteins for the limited pool of free $\beta$-cat

- Frizzled Frizzled proteins (11 different kinds in mammals) receptors with seven transmembrane domains. WNTs can bind the cysteine-rich domain of Frizzled, an extracellular part of the receptor

- LRP5/6 Low-density lipoprotein receptor-related proteins. They are co-receptors of the WNT ligands

- $S 33 Y \beta$-cat A constitutive active mutant of $\beta$-cat that is resistant to proteasome-mediated degradation

- $T C F$ There are four $\mathrm{T}$ cell factors (TCFs) in mammals, namely, TCF7 (also known as TCF-1), LEF-1, TCF7L1 (also known as TCF-3) and TCF7L2 (also known as TCF-4). In the absence of $\beta$-cat, a TCF protein binds to the consensus sequence (CTTTG[A/T][A/T]) in the WNT target gene promoters and represses their expression. $\beta$-Cat is able to convert a TCF protein into a transcriptional activator

- TCF7L2 Also known as TCF-4. Recent genome-wide association studies revealed that polymorphisms in $T C F 7 L 2$ have by far the biggest effect on the risk of type 2 diabetes

- WNT WNT glycoproteins (encoded by 19 different genes in mammals) function as ligands for the WNT signalling pathway. Although certain WNT ligands can stimulate c-Jun $\mathrm{NH}_{2}$-terminal kinase (JNK), calcium and protein kinase $\mathrm{C}$ signalling pathways, this review only discusses the role of the canonical WNT pathway, which uses the bipartite transcription factor $\beta$-cat/TCF as the major effector

\section{Introduction to the WNT signalling pathway}

The WNT signalling pathway (referred to as the WNT pathway hereafter) was initially recognised in colon cancer research and in embryonic development studies of the fruit fly (Drosophila melanogaster), frog (Xenopus laevis) and other organisms [1, 2]. Aberrant activation of the WNT pathway may lead to the development of colorectal and other types of tumours [3]. The major effector of the canonical
WNT pathway is the bipartite transcription factor $\beta$-catenin/ $\mathrm{T}$ cell factor $(\beta$-cat/TCF). This is formed by the heterodimerisation of free $\beta$-cat with one of the four members of the TCF family (TCF7 [also known as TCF-1], lymphoid enhancerbinding factor 1 [LEF-1], TCF7L1 [also known as TCF-3] and TCF7L2 [also known as TCF-4]), TCF7L2 being the major partner of $\beta$-cat in the intestinal epithelia $[3,4]$. As shown in Fig. 1a, in the absence of WNT, the cellular concentration of free $\beta$-cat is tightly controlled by a 'destructive complex', consisting of the tumour suppressor adenomatous polyposis coli (APC), axin, the serine threonine kinase glycogen synthase kinase-3 (GSK-3), casein kinase- $1 \alpha(\mathrm{CK}-1 \alpha)$ and phosphorylated ERK (pERK) [5, 6]. This complex interacts with $\beta$-cat and phosphorylates it. The phosphorylated $\beta$-cat is then destroyed via the proteasome-mediated degradation process. The WNT ligands exert their effect via the Frizzled receptors and the LDL receptor-related protein 5/6 (LRP5/6) co-receptors (Fig. 1b). Following receptor binding, WNT signals are transmitted by the association of WNT receptors with Dishevelled protein (Dvl). This event triggers the disruption of the destructive complex, preventing the phosphorylationdependent degradation of $\beta$-cat [4], which then enters the nucleus and interacts with a member of the TCF family to form a complex that stimulates the $\beta$-cat/TCF (or WNT) downstream target genes (Fig. 1b).

Many in vitro and in vivo examinations have shown that several components of the WNT pathway are involved in pancreatic beta cell proliferation [7-9], normal cholesterol metabolism and glucose-induced insulin secretion [10] and the production of the incretin hormone glucagon-like peptide-1 (GLP-1) [4, 11-13]. More recently, extensive genome-wide association (GWA) studies have identified TCF7L2 as a type 2 diabetes susceptibility gene. Of all the polymorphisms studied to date, TCF7L2 polymorphisms have been demonstrated to have by far the biggest effect on the risk of developing type 2 diabetes [14-20]. In addition, the human LRP5 gene was mapped to within the IDDM4 region, which is linked to type 1 diabetes on chromosome 11q13 [21-23]. Polymorphisms in LRP5 have been shown to be associated with obesity phenotypes, and missense mutations in LRP6 have been shown to be associated with the risk of bone loss, early coronary disease and the metabolic syndrome $[24,25]$. Moreover, polymorphisms in Wnt5B have been shown to be associated with the risk of type 2 diabetes [26].

The WNT pathway co-receptors (LRP5/6), a WNT ligand (WNT5B), and the major component of the WNT pathway effector (TCF7L2), are evidently involved in preventing the development of type 2 diabetes and other metabolic diseases 
a

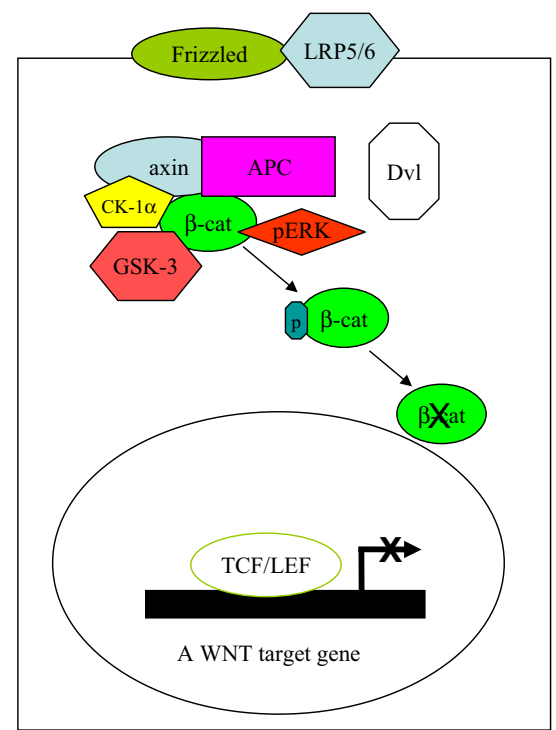

Fig. 1 Summary of the canonical WNT pathway. a In the absence of WNT stimulation, $\beta$-cat is located within the 'destructive complex', phosphorylated by GSK-3, CK- $1 \alpha$ and pERK, and subsequently destroyed by proteasome-mediated protein degradation. b Following WNT stimulation, the phosphorylation/destructive complex disassem-

In this review, I will first discuss the laboratory experimental studies on the role of the WNT pathway in the development/ genesis of mouse pancreatic islets, pancreatic beta cell growth and the production of the incretin hormone glucagon-like peptide-1 (GLP-1). This will be followed by a brief summary of GWA studies of TCF7L2 and the risk of type 2 diabetes. Finally, I will discuss recent findings indicating that forkhead box transcription factor subgroup $\mathrm{O}$ (FOXO) and TCF proteins are able to compete for the limited pool of $\beta$-cat, and ageing will lead to increased FOXO-mediated gene transcription and reduced TCF-mediated gene transcription. These findings give us new insights into type 2 diabetes as an age-dependent disease.

\section{WNT signalling is involved in the genesis of pancreatic islets and the proliferation of pancreatic beta cells}

Investigations into how $\beta$-cat, the major effector of WNT signalling, influences pancreatic islet development using transgenic and knockout mice has produced inconsistent results. Although several WNT ligands and the Frizzled receptors were shown to be produced in the embryonic and postnatal pancreas [27-29], an early study showed that the loss of $\beta$-cat did not significantly perturb pancreatic islet endocrine cell mass or function, although $\beta$-cat is essential for pancreatic exocrine acinar cell development [30]. Utilising the pancreatic and duodenal homeobox protein 1 (Pdx1)-Cre system to specifically delete the gene encoding $\beta$-cat $(C t n n b 1)$ in the epithelium of the pancreas and

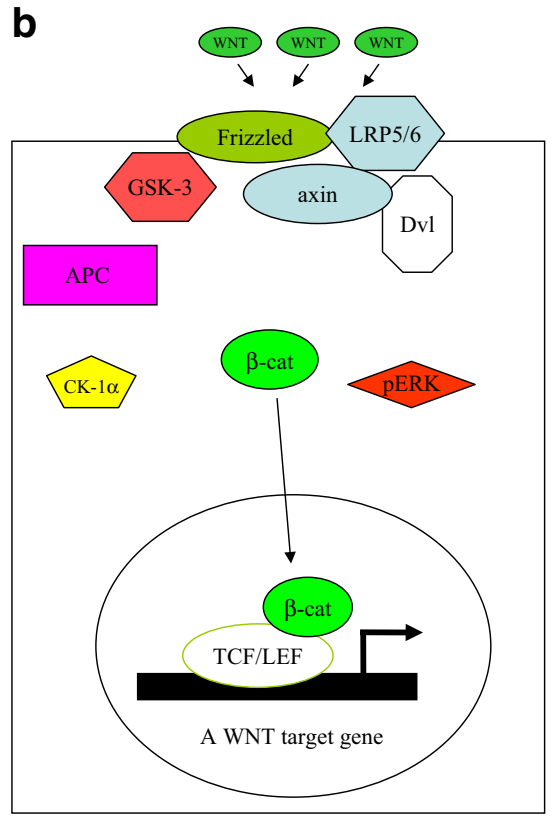

bles. This results in an accumulation of free $\beta$-cat, which enters the nucleus and forms the bipartite transcription factor $\beta$-cat/TCF, leading to enhanced expression of the WNT target genes. APC, adenomatous polyposis coli; pERK, phosphorylated ERK

duodenum revealed that Ctnnbl-deleted cells had a competitive disadvantage during pancreas development [31]. Although there was a reduction in endocrine islet numbers during early embryonic development and the mice developed pancreatitis perinatally because of the disruption of acinar epithelial structure, the mice later recovered from the pancreatitis and regenerated normal pancreas and duodenal villi from the wild-type cells that escaped the Ctnnb1 deletion [31]. However, a more recent study found that inducing the production of a stabilised form of $\beta$-cat (a mutant one that is more resistant to the proteasome-mediated degradation process) at different stages of development has different effects. During the early stage of organogenesis, robust production of stabilised $\beta$-cat drives changes in Hedgehog and fibroblast growth factor signalling and blocks the expression of the gene encoding Pdx-1, an important homeodomain protein transcription factor involved in the genesis of pancreatic beta cells. Induction of the stabilised form of $\beta$-cat at a later time point in pancreas development enhances proliferation and increases the size of this organ [32].

Seemingly contradictory results can be resolved if we assume that $\beta$-cat and $\beta$-cat/TCF exert different functions in a very precise dose-dependent manner at different developmental stages. It appears that mouse embryo is able to overcome a substantial reduction in $\beta$-cat via complicated compensatory mechanisms, which allow the generation of a normal pancreas 
Rulifson et al. recently examined the effect of WNT signalling in regulating beta cell genesis and proliferation using both in vitro and in vivo approaches [7]. Purified WNT3a (which is known to activate the canonical WNT pathway) stimulated proliferation of both the mouse beta cell line MIN6 and primary mouse pancreatic beta cells, possibly through the cell cycle regulators cyclin D1, cyclin D2 and cyclindependent kinase 4 , as well as the homeodomain transcription factor Pitx2. Immunohistological examinations of 3-month-old bi-transgenic rat insulin I promoter (RIP)-Cre and $\beta$-cat ${ }^{\text {active }}$ mice revealed a threefold increase in the production of Ki67 by pancreatic beta cells, which occurred in parallel with a 2.5-fold increase in beta cell mass [7]. Furthermore, axin production led to impaired Pitx2 gene expression, along with impaired beta cell expansion [7]. Taken together, these observations suggest that WNT signalling is necessary and sufficient for pancreatic beta cell proliferation.

The incretin hormone GLP-1 has been shown to stimulate WNT activity in adult mouse pancreatic islets, and both TCF7L2 and $\beta$-cat are required for GLP-1stimulated proliferation of the rat pancreatic beta cell line INS-1 [9]. Furthermore, adipocyte-derived WNT molecules have been reported to induce beta cell proliferation and insulin secretion in vitro [33].

In vivo mouse and in vitro cell line studies have revealed the essential function of WNT signalling in pancreatic beta cells during embryonic developmental stages and in adulthood

\section{The WNT co-receptor LRP5 is essential for normal lipid metabolism and glucose-induced insulin secretion}

Besides the effectors of WNT signalling, the co-receptors of the WNT ligands, LRP-5/6 [34, 35], are also important for normal lipid and glucose metabolism. An early study demonstrated that loss of function mutations of LRP5 were associated with the development of the autosomal recessive disorder osteoporosis-pseudoglioma syndrome [36]. The co-receptor was shown to be important in transducing WNT signalling and to play critical roles in modulating bone accrual and eye development [37]. The human LRP5 gene maps within the IDDM4 region on chromosome 11q13, which is linked to type 1 diabetes [21-23]. A recent GWA study has also shown that polymorphisms in LRP5 are associated with obesity phenotypes [38]. Pancreatic production of LRP5 has been reported [39, 40].

It has been demonstrated that LRP5 can interact with axin, one of the inhibitors of the WNT pathway (Fig. 1).
When Lrp5 was expressed in fibroblast cells, the LRP5 protein alone exerted no effect on the WNT pathway, but acted synergistically with the WNT ligands [41]. Furthermore, LRP5 molecules without the extracellular domain were constitutively active. They induced TCF/LEF-mediated transcription and stabilised $\beta$-cat [41]. The addition of WNT ligands to the medium triggered the translocation of axin to the cell membrane and enhanced the interaction between axin and LRP5. Finally, the LRP5 domain involved in the interaction with axin is also required for TCF/LEF-mediated transcriptional activation. These observations collectively suggest that binding of axin by LRP5 and its translocation to the cell membrane is an important part of WNT signal activation [41] (Fig. 1b).

After being fed a high-fat diet, $\operatorname{Lrp} 5^{-/-}$mice showed increased plasma cholesterol levels and after being fed a normal diet they showed markedly impaired glucose tolerance [10]. Furthermore, in response to the administration of high concentrations of glucose, the mice showed significant reductions in intracellular ATP and calcium levels and decreased glucose-induced insulin secretion [10]. The WNT ligands WNT3a and WNT5a stimulated insulin secretion in the wild-type mice but not in the $L R P 5^{-/-}$mice [10], suggesting that WNT ligands require a functional LRP5 to regulate insulin secretion.

In mice, functional LRP5 is important for normal lipid and glucose metabolism. In humans, mutations in LRP5 may result in obesity, type 1 diabetes and other metabolic diseases

\section{Both WNT and insulin pathways are involved in the production of the incretin hormone GLP-1}

GLP-1 is an important incretin hormone that is encoded by the Gcg gene, which is expressed in the intestinal endocrine L cells [42-44]. In these cells, expression of Gcg mRNA and production of GLP-1 can be activated by lithium, which mimics the function of the WNT ligands [45], or the overproduction of the constitutively active S33Y $\beta$-cat mutant [12], indicating that $G c g$ is a downstream target of the WNT signalling pathway. Activation was subsequently attributed to a TCF binding site within the G2 enhancer element of the $G c g$ promoter and the production of TCF7L2 in the intestinal endocrine L cells [4]. It is well known that Gcg expression and GLP-1 production can be activated by elevations in cAMP levels [46-52]. Since the G2 enhancer element has been shown to mediate the stimulatory effect of both cAMP and calcium on $\mathrm{Gcg}$ promoter activity [53], it is 
possible that cAMP pathway cross-talks with the WNT pathway to regulate $G c g$ expression [12].

Insulin inhibits $G c g$ expression in pancreatic alpha cells $[54,55]$. This inhibition is physiologically important because Gcg mRNA expression in pancreatic islets leads to the production of glucagon, the primary counter-regulatory hormone of insulin [54, 55]. A recent study showed a significant stimulatory effect of insulin on $G c g$ mRNA expression and GLP-1 production in intestinal L cells. Interestingly, insulin activated a $G c g$-luciferase (LUC) reporter gene construct containing the wild-type TCF binding site within the $\mathrm{G} 2$ enhancer element of the $G c g$ promoter, but not the one carrying a mutation at this site. Either 'knockingdown' $\beta$-cat production or the function of TCF7L2 completely blocked insulin-stimulated intestinal $G c g$ expression. Thus, insulin, at least partially uses effectors of the WNT pathway to exert this stimulatory effect [13]. Interestingly, the stimulatory effect of insulin on Gcg promoter activity was blocked by phosphatidylinositol 3-kinase (PI3K) inhibition, but not by protein kinase $\mathrm{B}$ (PKB) inhibition [13]. This, along with the observations of the existence of PKB-independent PI3K activity in other cell lineages [56-58], suggest that an unknown signalling component mediates PI3K-mediated cross-talk between insulin and WNT signalling pathways [13]. In hyperinsulinaemic and insulin-resistant MKR mice $[59,60]$, Gcg mRNA expression and GLP-1 production in the distal ileum were significantly higher than in sex- and age-matched controls [13], indicating that hyperinsulinaemia and/or insulin resistance may affect the homeostasis of GLP-1 production. Figure 2 shows that both WNT and cross-talk between insulin and WNT are involved in GLP-1 production, although detailed mechanisms and the pathophysiological significance of the cross-talk need to be further explored. Figure 2 also shows that lithium and cAMP may stimulate $G c g$ expression by affecting the production of free $\beta$-cat.

It appears that $\beta$-cat/TCF functions as the effector of other signalling pathways to regulate $G c g$ expression and GLP-1 production. It is likely that cross-talk between insulin and WNT pathways is involved in the homeostasis of GLP-1 production

\section{$T C F 7 L 2$ polymorphisms are associated with the risk of type 2 diabetes}

In 2006, a study reported that inheritance of specific single nucleotide polymorphisms (SNPs) within the TCF7L2 gene was related to an increased risk of type 2 diabetes [20]. The investigators of this study have genotyped 228 microsatellite

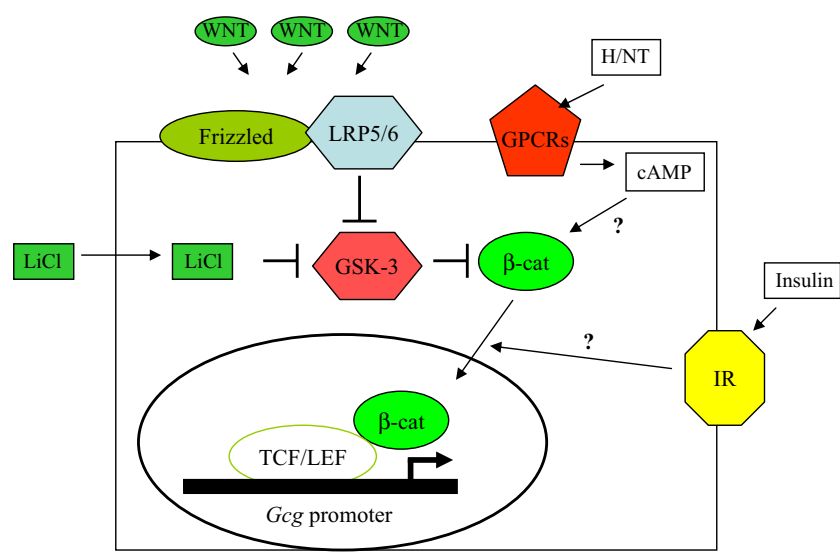

Fig. 2 Multiple signalling cascades utilise $\beta$-cat/TCF as the effector for the regulation of $\mathrm{Gcg}$ expression. Both WNT and $\mathrm{LiCl}$ increase levels of free $\beta$-cat through inhibition of GSK-3. Hormone and neurotransmitters $(\mathrm{H} / \mathrm{NT})$ may enhance $\beta$-cat/TCF activity by interacting with their G-protein-coupled receptors (GPCRs). Insulin may enhance the translocation of $\beta$-cat into the nucleus. The question marks indicate that detailed mechanisms for these events are still under investigation. IR, insulin receptor

markers in Icelandic individuals with type 2 diabetes and healthy controls across a $10.5 \mathrm{Mb}$ interval on chromosome 10q. A microsatellite, DG10S478, located within the intron 3 region of $T C F 7 L 2$, was found to be associated with type 2 diabetes [20]. This observation was subsequently replicated in both a Danish and US cohort [20]. The investigators found that two of five SNPs investigated within introns 4 and 5 of TCF7L2, namely rs12255372 and rs7903146, were in strong linkage disequilibrium with DG10S478 and showed similarly robust associations with type 2 diabetes [20]. This discovery has drawn attention globally [15-19, 61-71], and studies in many other ethnic groups have confirmed that rs12255372 and rs7903146 are the two SNPs most strongly associated with type 2 diabetes [72, 73], with the SNP rs7903146 reported to have the greatest effect in white individuals [73, 74]. The two SNPs occur at relatively low frequencies in Asian populations, although an association with type 2 diabetes was identified in two large Japanese cohorts [75, 76]. Recent studies have revealed two novel SNPs associated with the risk of type 2 diabetes. The SNP rs290487 was identified in a study of a Han Chinese population in Taiwan [77], and the SNP rs11196218 was identified in a study of Hong Kong Chinese individuals [78]. It has been reported that non-diabetic carriers of the risk-associated TCF7L2 SNPs do not have defects in GLP-1 secretion [64].

All the SNPs identified to date in $T C F 7 L 2$ are located within the intron regions of the gene. How these SNPs affect the expression of $T C F 7 L 2$, and therefore the risk of type 2 diabetes, remains largely unknown 
Since TCF7L2 is known as an intestinal cell specific transcription factor [79] and is an important regulator of intestinal $\mathrm{Gcg}$ expression and GLP-1 production [11], it was suggested that the TCF7L2 SNPs may modify disease susceptibility by affecting intestinal $G c g$ expression and plasma levels of GLP-1 [20]. More recently, genotyping 1,100 non-diabetic German individuals for the five known TCF7L2 SNPs indicated that TCF7L2 variants are associated with reduced insulin secretion [64]. In contrast, plasma GLP-1 levels during an OGTT were not significantly influenced by the TCF7L2 variants [64]. The CT/TT genotypes of the SNP rs7903146 were shown to strongly predict future type 2 diabetes in two independent Scandinavian cohorts [63]. The risk $\mathrm{T}$ allele was associated with impaired insulin secretion, incretin effects, and an enhanced rate of hepatic glucose production [63]. Furthermore, investigators found that islet TCF7L2 expression was increased fivefold in individuals with the TT genotype. Although TCF7L2 expression was positively correlated with the expression of INS, which encodes insulin, it was inversely correlated with glucose-stimulated insulin release [63]. Furthermore, an ex vivo examination demonstrated that TCF7L2 knockdown (with small interfering RNA) increased human pancreatic beta cell apoptosis and reduced beta cell proliferation and glucose-stimulated insulin secretion [8]. Overexpression of $T C F 7 L 2$, on the other hand, protected islets from glucose- and cytokine-induced apoptosis and from impaired functions [8]. As discussed above, both TCF7L2 and $\beta$-cat are required as effectors for GLP-1-stimulated beta cell proliferation [9].

Although these recent studies indicate that TCF7L2 SNPs may directly affect INS expression and/or insulin secretion, we still do not have a clear picture of how these SNPs affect the function of pancreatic beta cells. As discussed by Schafer and colleagues, the involvement of changes in GLP-1 production and secretion influenced by TCF7L2 variants in the increased risk of type 2 diabetes cannot be eliminated [64]. It should also be pointed out that the participants in their study were non-diabetic individuals. It is possible that a certain compensatory response(s) attenuated the defect in GLP-1 secretion in these TCF7L2 SNP carriers in the pre-diabetic stages.

\section{FOXOs compete with TCFs for the limited pool of $\beta$-cat}

Over the last few years, investigations have led to the discovery of the insulin-FOXO protein signalling cascade [80-83]. This regulatory system controls metabolic homeostasis and other important physiological and pathophysiological events. In the absence of insulin or growth factors, FOXOs are mainly located within the nuclei and upregulate the expression of a set of target genes, thereby promoting cell cycle arrest, stress resistance and apoptosis (Fig. 3a). In the presence of insulin or growth factors, FOXOs are phosphorylated by PKB and serum- and glucocorticoidregulated protein kinase and stay in the cell cytosol (Fig. 3b) [81]. In this way, FOXOs function to control the growth, development, metabolism, and possibly, longevity of the cell and the organism in response to insulin, insulinlike growth factor-1 and many other growth factors [81]. In contrast to the effect on insulin signalling, oxidative stress induces the activation of FOXO signalling [84]. It appears that this is due to the activation of the small GTPase Ral, which leads to the c-Jun $\mathrm{NH}_{2}$-terminal kinase (JNK)dependent phosphorylation of FOXOs, followed by their translocation to the nucleus and increased FOXO-mediated transcriptional activities [84].

An evolutionarily conserved interaction between the WNT pathway effector $\beta$-cat and FOXOs was discovered in 2005 [85]. In mammalian cells, a yeast two-hybrid screen detected an interaction between $\beta$-cat and FOXO1 and FOXO3. The gene bar-1 (also known as C54D1.6) encodes the Caenorhabditis elegans (nematode worm) homologue of $\beta$-cat [86], while the FOXO gene homologue in this organism is dafl6 [87]. An interaction between BAR-1 and DAF16 was also detected [85]. In mammalian cells, binding of $\beta$-cat to FOXO enhanced the transcriptional activity of FOXO. In C. elegans, the loss of BAR-1 reduced the activity of DAF16 in dauer formation and life span [85]. More importantly, the association between $\beta$-cat and FOXO was shown to be enhanced in cells exposed to oxidative stress [85].

The interaction between FOXO and $\beta$-cat prompted scientists to explore the pathophysiological role of this interaction in age-dependent diseases, including osteoporosis. It was reported that male and female mice with sufficient sex hormones could still lose bone mass and strength progressively during the ages of 4-31 months, and that this was associated with enhanced osteoblast and osteocyte apoptosis, reduced osteoblast number and bone formation rate, and elevated levels of reactive oxygen species (ROS) [88]. Furthermore, in the C57BL/6 mice, ageing was shown to be associated with the reduced expression of several WNT target gene mRNAs, including Axin2 and Opg (also known as Tnfrsfllb); and increased expression of the FOXO target genes, such as Gadd45 (also known as Gadd45a) [89]. However, hydrogen peroxide treatment in an uncommitted mesenchymal cell line, $\mathrm{C} 2 \mathrm{C} 12$, increased FOXO-mediated transcription and attenuated both basal and WNT3A-stimulated levels of Axin2 and other WNT target genes. Opposite effects of hydrogen peroxide on FOXO- and TCF-mediated transcription were confirmed by measuring the reporter gene activity of FOXO-LUC and TCF-LUC in response to different dosages of hydrogen peroxide. More recently, the concept of that the interaction 
a

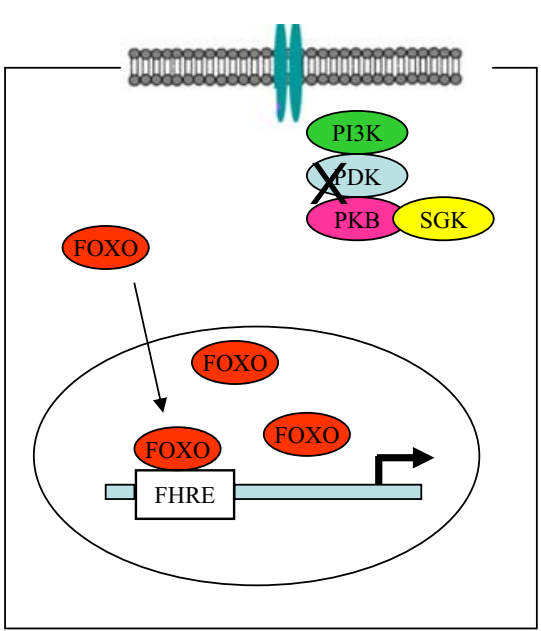

Cell cycle arrest, stress resistance and cell apoptosis

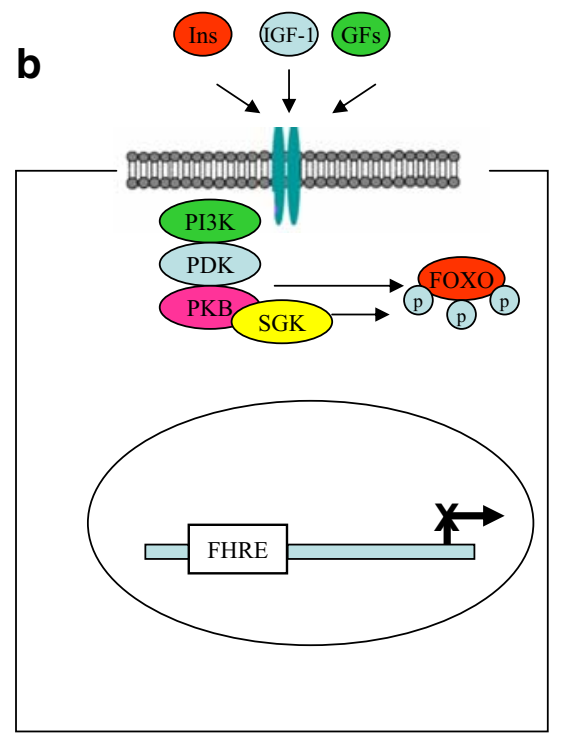

Cell proliferation, stress sensitive and cell survival
Fig. 3 Schematic representation of FOXO signalling. a Without insulin or a growth factor, FOXO enters the nucleus, binds to the forkhead response element (FHRE) of its downstream target genes. The activation of these genes (such as $p 27$ [also known as $C d k n 1 b$ ], Bim [also known as Bcl2l11], Gadd45 and Fasl) will lead to cell cycle arrest, stress resistance and cell apoptosis. b Insulin (Ins), insulin-like

of FOXO with $\beta$-cat inhibits $\beta$-cat/TCF activity was further confirmed [90]. It has been demonstrated that small interfering RNA (siRNA)-mediated knockdown of FOXO reverted the loss of $\beta$-cat binding to TCF after cellular growth factor-1 (IGF-1) and other growth factors (GFs) are able to activate the PI3K-PDK-1-PKB signalling pathway. Both PKB and serum- and glucocorticoid-inducible kinase (SGK) phosphorylate FOXOs, trapping them in the cytosol. PDK-1, 3'-phosphoinositidedependent kinase-1

oxidative stress [90]. The production and function of FOXO proteins in the pancreatic beta cells have been extensively investigated during the past few years [91]. It will be interesting to examine whether insulin and growth

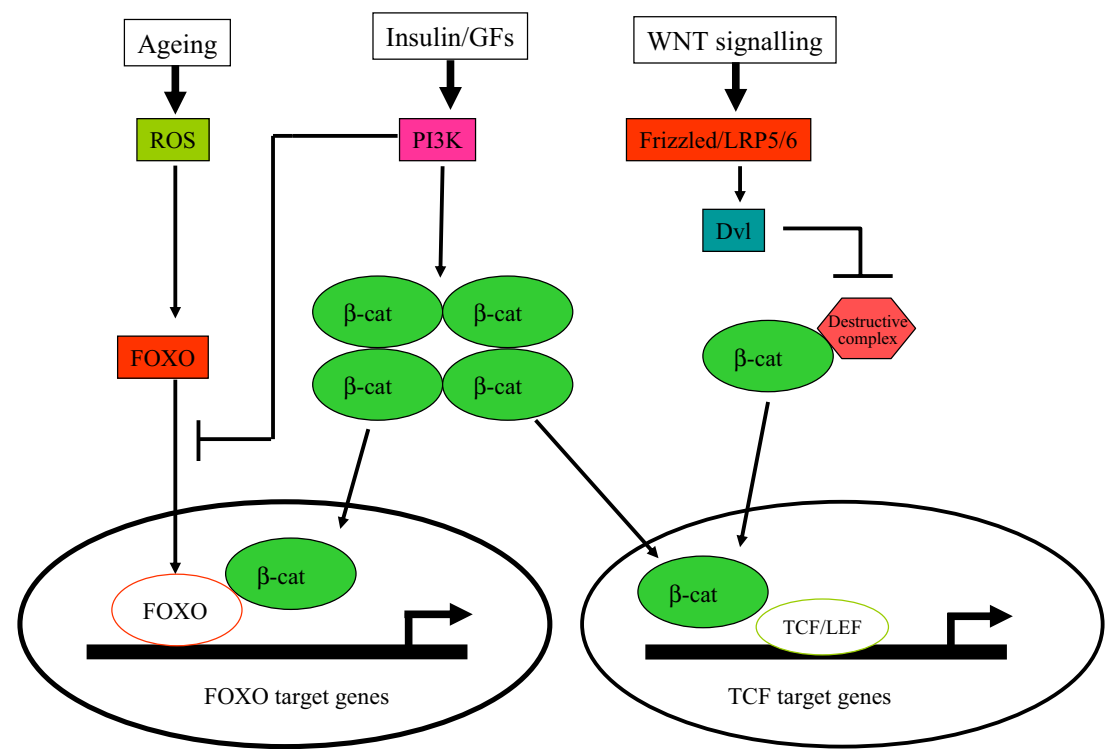

Fig. 4 Insulin/growth factors control the balance between FOXO- and TCF-mediated gene expression. FOXOs and TCFs compete for the limited pool of $\beta$-cat. During ageing and oxidative stress, the production of ROS leads to increased FOXO-mediated gene transcription and reduced TCF-mediated gene transcription. This will lead to reduced WNT activity, which is important for lipid and glucose metabolism, pancreatic beta cell proliferation and function and the production of the incretin hormone GLP-1. Insulin/growth factors (GFs) help to restore the balance by two means. First, they stimulate the nuclear exclusion of FOXOs via phosphorylation mediated by $\mathrm{PKB} /$ serum- and glucocorticoid-inducible kinase (SGK) [81, 82, 91]. Second, they enhance the nuclear content of $\beta$-cat and the binding of $\beta$-cat/TCF to the WNT target gene promoters via a yet to be identified PKB-independent mechanism [13] 
factors control the balance between FOXO-mediated and WNT-mediated gene transcription (see below).

FOXOs and TCF proteins compete for the common cofactor $\beta$-cat, while insulin/growth factors inhibit FOXOmediated gene expression by trapping FOXOs in the cell cytosol

\section{Summary and perspective}

Comprehensive in vitro and in vivo studies by multiple laboratories have shown that WNT signalling is important in normal pancreatic islet development, as well as in pancreatic beta cell function and genesis. It appears that the $\mathrm{WNT} / \beta$-cat pathway plays direct, precise, and even opposing roles during different stages of pancreatic islet development [32]. Obviously, to exert such precise and opposing effects, $W N T / \beta$-cat signalling needs to interact with other signalling pathways and regulate the downstream gene expression profiles, both temporally and spatially.

WNT signalling is also important in activating intestinal $G c g$ transcription and, therefore, the production of the incretin hormone GLP-1 [11-13], which has been shown to utilise WNT signalling effectors, i.e. $\beta$-cat/TCF7L2, to exert its effect on beta cell proliferation [9]. Further examinations are required to verify whether TCF7L2, certain WNT ligands and Frizzled receptors are also involved in the genesis of pancreatic islets and intestinal endocrine $\mathrm{L}$ cells.

GWA have revealed relationships between SNPs in LRP5, which encodes a co-receptor of the WNT ligands, and the risk of type 1 diabetes [22, 23], as well as obesity [29]. LRP6 mutations are possibly related to the development of bone loss, coronary disease and the metabolic syndrome [24, 25]. More importantly, extensive recent studies have identified associations between SNPs in TCF7L2 and the risk of type 2 diabetes. These observations suggest that WNT signalling is not only involved in pancreatic islet development during embryogenesis, but also in the function of pancreatic and intestinal endocrine cells during adulthood. Since all the known risk-associated SNPs of $T C F 7 L 2$ are located within the intronic regions, the effect of these SNPs on TCF7L2 expression should be examined. To ultimately understand why these SNPs affect the risk of type 2 diabetes, we need to explore mechanisms underlying TCF7L2 production in pancreatic and intestinal endocrine cells under both physiological and pathological conditions.

Type 2 diabetes is a chronic and age-dependent disease. As shown in Fig. 4, nuclear FOXOs increase during ageing because of the accumulation of ROS and JNK signalling pathway activation [92]. FOXOs compete with TCF proteins, including TCF7L2, for the limited pool of $\beta$-cat. This leads to reduced WNT activity, which is important for lipid and glucose metabolism, pancreatic beta cell proliferation and function, and the production of the incretin hormone GLP-1. Insulin and growth factors, on the other hand, may restore the balance between FOXO- and TCFmediated gene transcription by trapping FOXOs within the cell cytosol. The establishment of this concept offers a new perspective on the pathogenesis of type 2 diabetes and other age-dependent diseases. Further examination of the crosstalk between insulin/growth factors and WNT signalling pathways may lead to the development of novel therapeutic approaches for the treatment of type 2 diabetes and other age-dependent diseases.

Acknowledgements The author thanks Canadian Institutes of Health Research (CIHR grant no. 68991) and Banting and Best Diabetes Centre (BBDC) for supporting his research team in studying the role of WNT signalling in intestinal proglucagon gene expression and GLP-1 production. The author regrets not being able to cite all excellent contributions in the field because of space limitations.

Duality of interest The author declares that there is no duality of interest associated with this manuscript.

Open Access This article is distributed under the terms of the Creative Commons Attribution Noncommercial License which permits any noncommercial use, distribution, and reproduction in any medium, provided the original author(s) and source are credited.

\section{References}

1. Peifer M, Polakis P (2000) Wnt signaling in oncogenesis and embryogenesis - a look outside the nucleus. Science 287:16061609

2. Moon RT, Brown JD, Torres M (1997) WNTs modulate cell fate and behavior during vertebrate development. Trends Genet 13:157-162

3. Morin PJ, Sparks AB, Korinek V et al (1997) Activation of betacatenin-Tcf signaling in colon cancer by mutations in beta-catenin or APC. Science 275:1787-1790

4. Doble BW, Woodgett JR (2003) GSK-3: tricks of the trade for a multi-tasking kinase. J Cell Sci 116:1175-1186

5. Zeng X, Tamai K, Doble B et al (2005) A dual-kinase mechanism for Wnt co-receptor phosphorylation and activation. Nature 438: 873-877

6. Polakis P (2000) Wnt signaling and cancer. Genes Dev 14:18371851

7. Rulifson IC, Karnik SK, Heiser PW et al (2007) Wnt signaling regulates pancreatic beta cell proliferation. Proc Natl Acad Sci USA 104:6247-6252

8. Shu L, Sauter NS, Schulthess FT, Matveyenko AV, Oberholzer J, Maedler K (2008) TCF7L2 regulates cell survival and function in human pancreatic islets. Diabetes 57:645-653

9. Liu Z, Habener JF (2008) Glucagon-like peptide-1 activation of TCF7L2-dependent Wnt signaling enhances pancreatic beta-cell proliferation. J Biol Chem 283:8723-8735 
10. Fujino T, Asaba H, Kang MJ et al (2003) Low-density lipoprotein receptor-related protein 5 (LRP5) is essential for normal cholesterol metabolism and glucose-induced insulin secretion. Proc Natl Acad Sci USA 100:229-234

11. Yi F, Brubaker PL, Jin T (2005) TCF-4 mediates cell type-specific regulation of proglucagon gene expression by beta-catenin and glycogen synthase kinase-3beta. J Biol Chem 280:1457-1464

12. Ni Z, Anini Y, Fang X, Mills G, Brubaker PL, Jin T (2003) Transcriptional activation of the proglucagon gene by lithium and beta-catenin in intestinal endocrine $\mathrm{L}$ cells. J Biol Chem 278:1380-1387

13. Yi F, Sun J, Lim GE, Fantus IG, Brubaker PL, Jin T (2008) Crosstalk between the insulin and Wnt signaling pathways: evidence from intestinal endocrine L cells. Endocrinology 149:2341-2351

14. Weedon MN (2007) The importance of TCF7L2. Diabet Med 24:1062-1066

15. Owen KR, McCarthy MI (2007) Genetics of type 2 diabetes. Curr Opin Genet Dev 17:239-244

16. Elbein SC (2007) Evaluation of polymorphisms known to contribute to risk for diabetes in African and African-American populations. Curr Opin Clin Nutr Metab Care 10:415-419

17. Frayling TM (2007) A new era in finding type 2 diabetes genesthe unusual suspects. Diabet Med 24:696-701

18. Grarup N, Andersen G (2007) Gene-environment interactions in the pathogenesis of type 2 diabetes and metabolism. Curr Opin Clin Nutr Metab Care 10:420-426

19. Florez JC (2007) The new type 2 diabetes gene TCF7L2. Curr Opin Clin Nutr Metab Care 10:391-396

20. Grant SF, Thorleifsson G, Reynisdottir I et al (2006) Variant of transcription factor 7-like 2 (TCF7L2) gene confers risk of type 2 diabetes. Nat Genet 38:320-323

21. Hey PJ, Twells RC, Phillips MS et al (1998) Cloning of a novel member of the low-density lipoprotein receptor family. Gene 216:103-111

22. Twells RC, Mein CA, Payne F et al (2003) Linkage and association mapping of the LRP5 locus on chromosome 11q13 in type 1 diabetes. Hum Genet 113:99-105

23. Twells RC, Mein CA, Phillips MS et al (2003) Haplotype structure, LD blocks, and uneven recombination within the LRP5 gene. Genome Res 13:845-855

24. Mani A, Radhakrishnan J, Wang H et al (2007) LRP6 mutation in a family with early coronary disease and metabolic risk factors. Science 315:1278-1282

25. Kokubu C, Heinzmann U, Kokubu T et al (2004) Skeletal defects in ringelschwanz mutant mice reveal that Lrp6 is required for proper somitogenesis and osteogenesis. Development 131:5469-5480

26. Kanazawa A, Tsukada S, Sekine A et al (2004) Association of the gene encoding wingless-type mammary tumor virus integrationsite family member 5B (WNT5B) with type 2 diabetes. Am J Hum Genet 75:832-843

27. Heller RS, Dichmann DS, Jensen J et al (2002) Expression patterns of Wnts, Frizzleds, sFRPs, and misexpression in transgenic mice suggesting a role for Wnts in pancreas and foregut pattern formation. Dev Dyn 225:260-270

28. Heller RS, Klein T, Ling Z et al (2003) Expression of Wnt, Frizzled, sFRP, and DKK genes in adult human pancreas. Gene Expr 11:141-147

29. Lim HW, Lee JE, Shin SJ et al (2002) Identification of differentially expressed mRNA during pancreas regeneration of rat by mRNA differential display. Biochem Biophys Res Commun 299:806-812

30. Murtaugh LC, Law AC, Dor Y, Melton DA (2005) Beta-catenin is essential for pancreatic acinar but not islet development. Development 132:4663-4674

31. Papadopoulou S, Edlund H (2005) Attenuated Wnt signaling perturbs pancreatic growth but not pancreatic function. Diabetes $54: 2844-2851$
32. Heiser PW, Lau J, Taketo MM, Herrera PL, Hebrok M (2006) Stabilization of beta-catenin impacts pancreas growth. Development 133:2023-2032

33. Schinner S, Ulgen F, Papewalis C et al (2008) Regulation of insulin secretion, glucokinase gene transcription and beta cell proliferation by adipocyte-derived Wnt signalling molecules. Diabetologia $51: 147-154$

34. Tamai K, Semenov M, Kato Y et al (2000) LDL-receptor-related proteins in Wnt signal transduction. Nature 407:530-535

35. Wehrli M, Dougan ST, Caldwell K et al (2000) arrow encodes an LDL-receptor-related protein essential for Wingless signalling. Nature 407:527-530

36. Gong Y, Slee RB, Fukai N et al (2001) LDL receptor-related protein 5 (LRP5) affects bone accrual and eye development. Cell 107:513523

37. Kato M, Patel MS, Levasseur R et al (2002) Cbfa1-independent decrease in osteoblast proliferation, osteopenia, and persistent embryonic eye vascularization in mice deficient in Lrp5, a Wnt coreceptor. J Cell Biol 157:303-314

38. Guo YF, Xiong DH, Shen H et al (2006) Polymorphisms of the low-density lipoprotein receptor-related protein 5 (LRP5) gene are associated with obesity phenotypes in a large family-based association study. J Med Genet 43:798-803

39. Figueroa DJ, Hess JF, Ky B et al (2000) Expression of the type I diabetes-associated gene LRP5 in macrophages, vitamin A system cells, and the islets of Langerhans suggests multiple potential roles in diabetes. J Histochem Cytochem 48:1357-1368

40. Kim DH, Inagaki Y, Suzuki T et al (1998) A new low density lipoprotein receptor related protein, LRP5, is expressed in hepatocytes and adrenal cortex, and recognizes apolipoprotein E. J Biochem 124: 1072-1076

41. Mao J, Wang J, Liu B et al (2001) Low-density lipoprotein receptorrelated protein-5 binds to Axin and regulates the canonical Wnt signaling pathway. Mol Cell 7:801-809

42. Kieffer TJ (2004) Gastro-intestinal hormones GIP and GLP-1. Ann Endocrinol (Paris) 65:13-21

43. Drucker DJ (2006) The biology of incretin hormones. Cell Metab $3: 153-165$

44. Holst JJ (2007) The physiology of glucagon-like peptide 1 . Physiol Rev 87:1409-1439

45. Stambolic V, Ruel L, Woodgett JR (1996) Lithium inhibits glycogen synthase kinase-3 activity and mimics Wingless signalling in intact cells. Curr Biol 6:1664-1668

46. Drucker DJ, Jin T, Asa SL, Young TA, Brubaker PL (1994) Activation of proglucagon gene transcription by protein kinase-A in a novel mouse enteroendocrine cell line. Mol Endocrinol 8:1646-1655

47. Lu F, Jin T, Drucker DJ (1996) Proglucagon gene expression is induced by gastrin-releasing peptide in a mouse enteroendocrine cell line. Endocrinology 137:3710-3716

48. Chen L, Wang P, Andrade CF et al (2005) PKA independent and cell type specific activation of the expression of caudal homeobox gene Cdx-2 by cyclic AMP. FEBS J 272:2746-2759

49. Lotfi S, Li Z, Sun J et al (2006) Role of the exchange protein directly activated by cyclic adenosine 5 '-monophosphate (Epac) pathway in regulating proglucagon gene expression in intestinal endocrine L cells. Endocrinology 147:3727-3736

50. Drucker DJ, Campos R, Reynolds R, Stobie K, Brubaker PL (1991) The rat glucagon gene is regulated by a protein kinase Adependent pathway in pancreatic islet cells. Endocrinology 128:394-400

51. Gajic D, Drucker DJ (1993) Multiple cis-acting domains mediate basal and adenosine 3',5'-monophosphate-dependent glucagon gene transcription in a mouse neuroendocrine cell line. Endocrinology 132:1055-1062

52. Wang J, Cao Y, Steiner DF (2003) Regulation of proglucagon transcription by activated transcription factor (ATF) 3 and a 
novel isoform, ATF3b, through the cAMP-response element/ATF site of the proglucagon gene promoter. J Biol Chem 278:32899 32904

53. Furstenau U, Schwaninger M, Blume R, Jendrusch EM, Knepel W (1999) Characterization of a novel calcium response element in the glucagon gene. J Biol Chem 274:5851-5860

54. Philippe J (1989) Glucagon gene transcription is negatively regulated by insulin in a hamster islet cell line. J Clin Invest 84:672-677

55. Philippe J (1991) Insulin regulation of the glucagon gene is mediated by an insulin-responsive DNA element. Proc Natl Acad Sci USA 88:7224-7227

56. Sun J, Jin T (2008) Both Wnt and mTOR signaling pathways are involved in insulin-stimulated proto-oncogene expression in intestinal cells. Cell Signal 20:219-229

57. Zhang Q, Adiseshaiah P, Kalvakolanu DV, Reddy SP (2006) A phosphatidylinositol 3-kinase-regulated Akt-independent signaling promotes cigarette smoke-induced FRA-1 expression. J Biol Chem 281:10174-10181

58. Qiao M, Shapiro P, Kumar R, Passaniti A (2004) Insulin-like growth factor-1 regulates endogenous RUNX2 activity in endothelial cells through a phosphatidylinositol 3-kinase/ERK-dependent and Aktindependent signaling pathway. J Biol Chem 279:42709-42718

59. Fernandez AM, Kim JK, Yakar S et al (2001) Functional inactivation of the IGF-I and insulin receptors in skeletal muscle causes type 2 diabetes. Genes Dev 15:1926-1934

60. Asghar Z, Yau D, Chan F, Leroith D, Chan CB, Wheeler MB (2006) Insulin resistance causes increased beta-cell mass but defective glucose-stimulated insulin secretion in a murine model of type 2 diabetes. Diabetologia 49:90-99

61. Freathy RM, Weedon MN, Bennett A et al (2007) Type 2 diabetes TCF7L2 risk genotypes alter birth weight: a study of 24,053 individuals. Am J Hum Genet 80:1150-1161

62. Florez JC, Jablonski KA, Bayley N et al (2006) TCF7L2 polymorphisms and progression to diabetes in the Diabetes Prevention Program. N Engl J Med 355:241-250

63. Lyssenko V, Lupi R, Marchetti P et al (2007) Mechanisms by which common variants in the TCF7L2 gene increase risk of type 2 diabetes. J Clin Invest 117:2155-2163

64. Schafer SA, Tschritter O, Machicao F et al (2007) Impaired glucagonlike peptide-1-induced insulin secretion in carriers of transcription factor 7-like 2 (TCF7L2) gene polymorphisms. Diabetologia 50:2443-2450

65. Cauchi S, Choquet H, Gutierrez-Aguilar R et al (2008) Effects of TCF7L2 polymorphisms on obesity in European populations. Obesity (Silver Spring) 16:476-482

66. Cauchi S, El Achhab Y, Choquet H et al (2007) TCF7L2 is reproducibly associated with type 2 diabetes in various ethnic groups: a global meta-analysis. J Mol Med 85:777-782

67. Cauchi S, Meyre D, Choquet H et al (2007) TCF7L2 rs7903146 variant does not associate with smallness for gestational age in the French population. BMC Med Genet 8:37

68. Cauchi S, Meyre D, Choquet H et al (2006) TCF7L2 variation predicts hyperglycemia incidence in a French general population: the Data from an Epidemiological Study on the Insulin Resistance Syndrome (DESIR) study. Diabetes 55:3189-3192

69. Cauchi S, Meyre D, Dina C et al (2006) Transcription factor TCF7L2 genetic study in the French population: expression in human beta-cells and adipose tissue and strong association with type 2 diabetes. Diabetes 55:2903-2908

70. Cauchi S, Proenca C, Choquet $\mathrm{H}$ et al (2008) Analysis of novel risk loci for type 2 diabetes in a general French population: the D.E.S.I.R. study. J Mol Med 86:341-348

71. Duan QL, Dube MP, Frasure-Smith N et al (2007) Additive effects of obesity and TCF7L2 variants on risk for type 2 diabetes among cardiac patients. Diabetes Care 30:1621-1623
72. Saxena R, Gianniny L, Burtt NP et al (2006) Common single nucleotide polymorphisms in TCF7L2 are reproducibly associated with type 2 diabetes and reduce the insulin response to glucose in nondiabetic individuals. Diabetes 55:2890-2895

73. Helgason A, Palsson S, Thorleifsson G et al (2007) Refining the impact of TCF7L2 gene variants on type 2 diabetes and adaptive evolution. Nat Genet 39:218-225

74. Goodarzi MO, Rotter JI (2007) Testing the gene or testing a variant? The case of TCF7L2. Diabetes 56:2417-2419

75. Hayashi T, Iwamoto Y, Kaku K, Hirose H, Maeda S (2007) Replication study for the association of TCF7L2 with susceptibility to type 2 diabetes in a Japanese population. Diabetologia 50:980 984

76. Horikoshi M, Hara K, Ito C, Nagai R, Froguel P, Kadowaki T (2007) A genetic variation of the transcription factor 7-like 2 gene is associated with risk of type 2 diabetes in the Japanese population. Diabetologia 50:747-751

77. Chang YC, Chang TJ, Jiang YD, Kuo SS, Lee KC, Chiu KC, Chuang LM (2007) Association study of the genetic polymorphisms of the transcription factor 7-like 2 (TCF7L2) gene and type 2 diabetes in the Chinese population. Diabetes 56:2631-2637

78. Ng MC, Tam CH, Lam VK, So WY, Ma RC, Chan JC (2007) Replication and identification of novel variants at TCF7L2 associated with type 2 diabetes in Hong Kong Chinese. J Clin Endocrinol Metab 92:3733-3737

79. Korinek V, Barker N, Moerer P, van Donselaar E, Huls G, Peters PJ, Clevers H (1998) Depletion of epithelial stem-cell compartments in the small intestine of mice lacking Tcf-4. Nat Genet 19:379-383

80. Puig O, Tjian R (2006) Nutrient availability and growth: regulation of insulin signaling by dFOXO/FOXO1. Cell Cycle 5:503-505

81. Greer EL, Brunet A (2005) FOXO transcription factors at the interface between longevity and tumor suppression. Oncogene 24:7410-7425

82. Accili D, Arden KC (2004) FoxOs at the crossroads of cellular metabolism, differentiation, and transformation. Cell 117:421-426

83. Barthel A, Schmoll D, Unterman TG (2005) FoxO proteins in insulin action and metabolism. Trends Endocrinol Metab 16:183-189

84. Essers MA, Weijzen S, de Vries-Smits AM et al (2004) FOXO transcription factor activation by oxidative stress mediated by the small GTPase Ral and JNK. EMBO J 23:4802-4812

85. Essers MA, de Vries-Smits LM, Barker N, Polderman PE, Burgering BM, Korswagen HC (2005) Functional interaction between beta-catenin and FOXO in oxidative stress signaling. Science 308:1181-1184

86. Eisenmann DM, Maloof JN, Simske JS, Kenyon C, Kim SK (1998) The beta-catenin homolog BAR-1 and LET-60 Ras coordinately regulate the Hox gene lin-39 during Caenorhabditis elegans vulval development. Development 125:3667-3680

87. Tissenbaum HA, Guarente L (2002) Model organisms as a guide to mammalian aging. Dev Cell 2:9-19

88. Almeida M, Han L, Martin-Millan M et al (2007) Skeletal involution by age-associated oxidative stress and its acceleration by loss of sex steroids. J Biol Chem 282:27285-27297

89. Almeida M, Han L, Martin-Millan M, O’Brien CA, Manolagas SC (2007) Oxidative stress antagonizes Wnt signaling in osteoblast precursors by diverting beta-catenin from $\mathrm{T}$ cell factor- to forkhead box O-mediated transcription. J Biol Chem 282:27298-27305

90. Hoogeboom D, Essers MA, Polderman PE, Voets E, Smits LM, Burgering BM (2008) Interaction of FOXO with beta-catenin inhibits beta-catenin/TCF activity. J Biol Chem 283:9224-9230

91. Glauser DA, Schlegel W (2007) The emerging role of FOXO transcription factors in pancreatic beta cells. J Endocrinol 193:195207

92. Nishikawa T, Araki E (2007) Impact of mitochondrial ROS production in the pathogenesis of diabetes mellitus and its complications. Antioxid Redox Signal 9:343-353 